\title{
Comparative Analysis of Higher Order Modulation Schemes using Simulink
}

\author{
P. Naga Malleswari \\ Department of ECE, CVR College of Engineering, Hyderabad, India \\ Email: pinjalamalleswari@gmail.com.
}

\begin{abstract}
There is always a search for digital modulation technique that has efficient bandwidth and low bit error rate at a relatively low signal to noise power. Considering this requirement, in this paper we provide a general theoretical analysis of various M-ary PSK modulation for $M=2,4,8,16$, and 32, $M$-ary $Q A M$ for $M=4,16$ and 64 and $M-$ aryDPSK for $\mathrm{M}=2$ and 4 using MATLAB Simulink taking BER as measure of performance when the system is subjected to Additive White Gaussian Channel. In this paper we take up bandwidth efficient linear digital modulation schemes and compare its performance based on their theoretical BER over AWGN and proves that increasing the value of $M$ results in increase of BER with the increase in data rate.
\end{abstract}

Index terms - Bit error Rate (BER),Additive White Gaussian Noise (AWGN), M-ary PSK, M-ary QAM, M-ary DPSK and Signal-to Noise Ratio (SNR).

\section{INTRODUCTION}

In digital communications industry the latest mathematical softwares can be used to increase the performance of digital system with several modulation techniques in digital like ASK (Amplitude Shift Keying), FSK(Frequency Shift Keying), PSK(Phase Shift Keying) PSK and QAM (Quadrature Amplitude Modulation) schemes. This paper contains both coherent and non coherent detection schemes. In later scheme, prior knowledge of the channel impulse response is unknown at the receiver. The phase of the received signal $y$ is uniformly distributed between 0 and 2pi.This signaling scheme fails completely in the absence of noise. So this scheme is not a suitable method of detection especially in a fading environment. In coherent detection the receiver has sufficient knowledge about the channel impulse response. Pilot transmission technique is used to estimate the channel impulse response at the receiver. Equalization process gives the desired transmitted symbols (' $x$ ') from the receiver signal (' $y$ ') by using the following equation.

$\hat{y}=\frac{y}{h}=\frac{h x+n}{h}=x+z$

Where $\mathrm{z}$ is an AWGN noise except for the scaling factor $1 / \mathrm{h}$.

\section{A. Bit Error Rate (BER)}

Due to noise, distortion, interference, or bit synchronization errors are introduced in Digital transmission scheme. The Bit Error Rate (BER) is the number of bits in error divided by the total number of transferred bits during a studied time interval. BER is a unit less performance measure. The bit error rate $\mathrm{Pb}$ is given by

$$
\text { Bit Error Rate }\left(\mathrm{P}_{\mathrm{b}}\right)=\frac{\text { Number of bits in error }}{\text { Total number of transfered bits }}
$$

Each modulation scheme performance can be measured by using probability of error with additive white gaussian noise. High data rate like 16-PSK can transmit four bits per symbol. To achieve a low BER, high power is not required. Modulation schemes are capable of delivering more bits per symbol are more immune to errors caused by noise and interference in the channel in communication system. Due to the increase of number of users, the mobile terminal is subjected to mobility. Thus the application of higher order modulations becomes a hot topic for many researches.

\section{B. AWGN (Additive White Gaussian Noise) Channel}

Generally noise is an unwanted signal presents in systems and the term additive indicates the noise is superimposed to the signal that tends to corrupt the signal where it will limit the rate of message transmission and limit the receiver ability to make correct symbol decisions [1].The received signal is the combination of transmitted and AWGN noise. The mathematical equation for Gaussian probability distribution function $\mathrm{p}(\mathrm{z})$ can be written as

$$
p(z)=\frac{1}{\sigma \sqrt{2 \pi}} \exp \left[-\frac{1}{2}\left(\frac{z-a}{\sigma}\right)^{2}\right]
$$

Thus, AWGN is generated by thermal motion of electron in all dissipative electrical components like resistors, wires and so on. Mathematically, this noise is described by a zero-mean Gaussian random process where the random signal $(\mathrm{z})$ is a sum of Gaussian noise random variable (n) and a dc signal (a) that is

$$
z=a+n
$$

Thermal noise Power Spectral Density (PSD) is flat for all frequencies and is represented as

$$
G_{n}(f)=\frac{N_{0}}{2}
$$


Equation (5) gives power spectral density with two sides equally distributed for both positive and negative frequencies [1]. When noise power has a uniform spectral density, then it is called as white noise. Since thermal noise is present in all communication systems with additive, white and Gaussian characteristics to model the noise in communication systems.

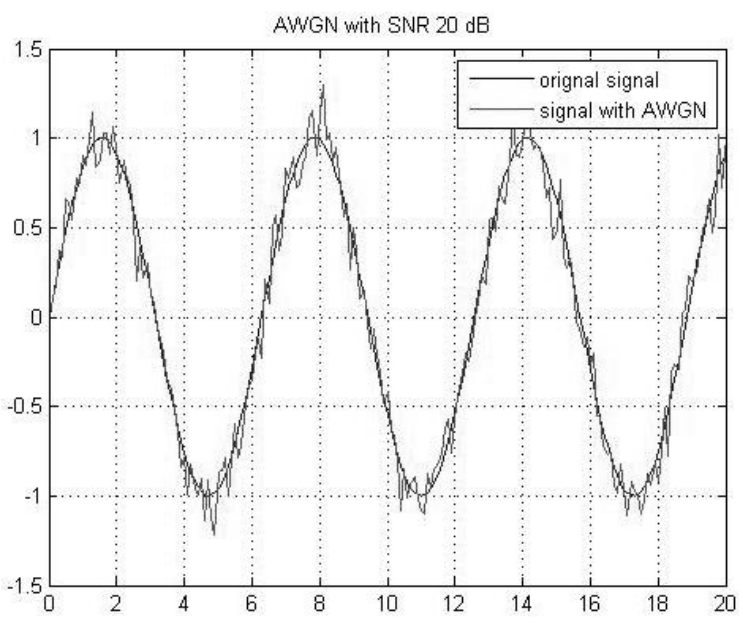

Fig.1: Example of AWGN signal

\section{Digital Modulation Techniques}

\section{A. M-ARY PSK Modulation}

In M-ary PSK BPSK ,QPSK ,8PSK,16PSK and 32PSK can be obtained by substituting $\mathrm{M}=2,4,8,16$, and 32 .

BPSK is the simplest forms of PSK techniques which gives better performance compared to ASK and FSK. In this modulation, information is conveyed by varying the phase of constant amplitude carrier signal between two states which are separated by 180 degrees, i.e., the phase of carrier changes between 0 and 180degrees.

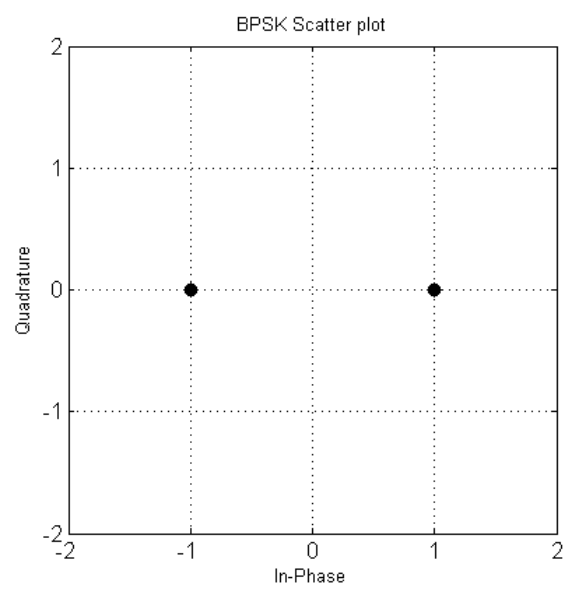

Fig.2: Constellation Diagram for BPSK

From Figure. 2 symbols represents the constellation points of the signal. BPSK modulated signal is given by $S_{1}(t)=A \cos \left(2 \pi f_{c} t\right) 0 \leq t \leq T$ for 1 ,
$S_{1}(t)=-A \cos \left(2 \pi f_{c} t\right) 0 \leq t \leq T$ for 0,

these two signals have the same frequency and energy. BPSK bandwidth efficiency is more but low compared to other Mary PSK techniques. Low symbol rate of BPSK is not suitable for high data rate applications. This is mainly used for deep space telemetry. To avoid spectral spreading filtering can be employed.

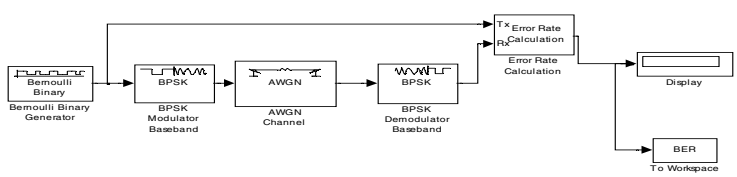

Fig. 3: BPSK Simulink Model

Figure. 3 shows the Simulink based Binary Phase Shift Keying. In this model we used Bernoullis Binary data Generator,BPSK Modulator and Demodulator, Additive White Gaussian Noise Channel and Error Rate Calculation Blocks. All these block are collected from MATLAB Simulink Communication Block Set.

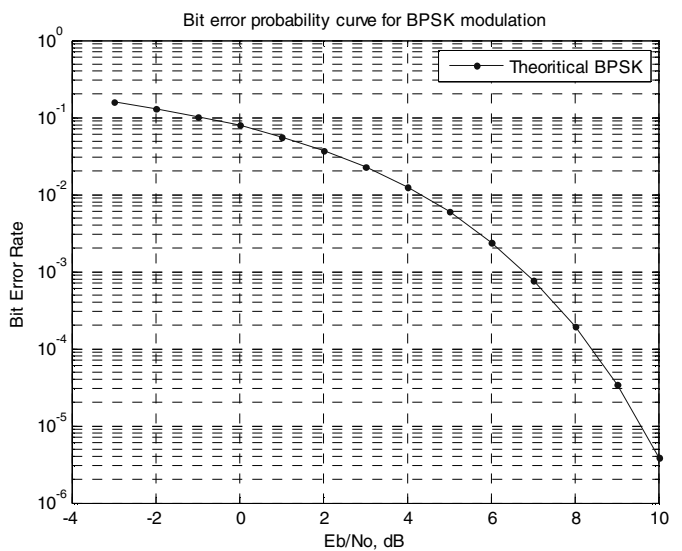

Fig.4: Bit error rate probability for BPSK over AWGN

Figure.4 shows the performance analysis of BPSK modulation over AWGN channel. It shows that the probability of error Vs Eb/No for BPSK.

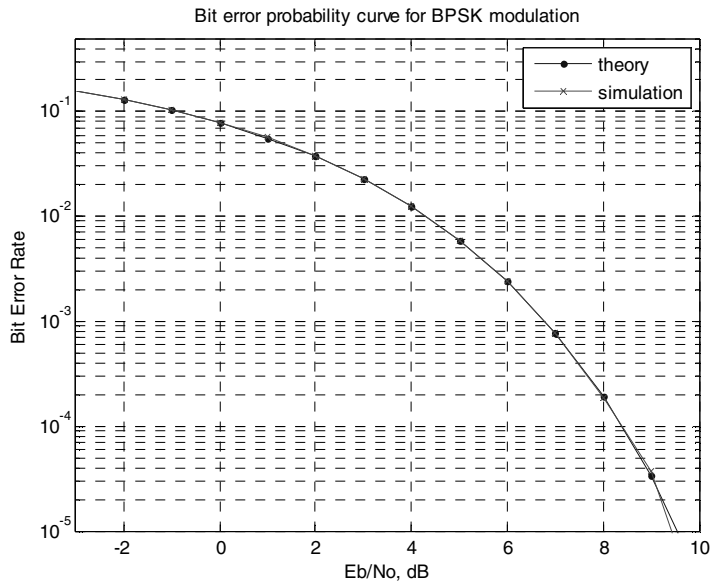

Fig.5: Theoritical vs Simulated analysis of BPSK over AWGN Channel using Simulink. 
Figure.5 shows that the comparative analysis of theoretical and simulated Bit error rate for BPSK modulation over Additive White Gaussian Noise channel.

\section{B. Quadrature Phase Shift Keying (QPSK)}

QPSK is widely used in different applications as it does not suffer BER degradation. Like BPSK, information in QPSK is conveyed by shifting the phase of carrier.

The only difference is, the phase of carrier in this case is shifted between four different states separated by 90 degrees each. It has 4 states, two states are inphase component values and other two are quadrature component values.

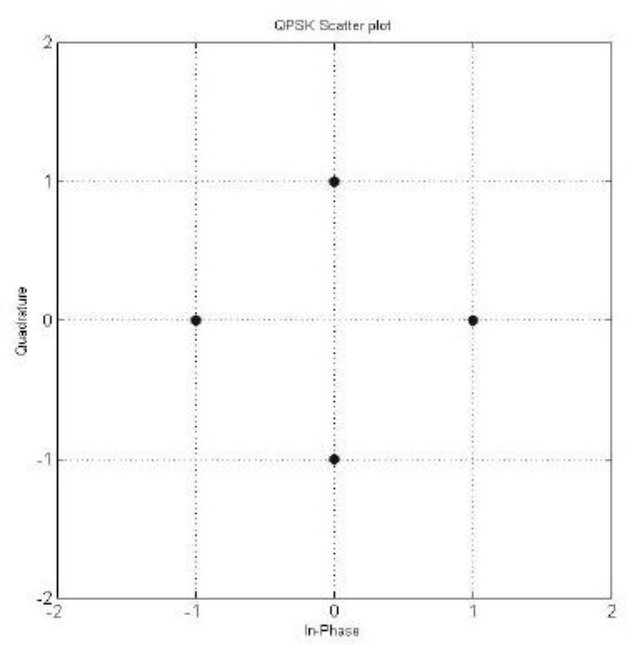

Fig.6: QPSK constellation diagram

Figure. 6 shows that 4 constellation points with a phase difference of 90 degrees and equispaced around a circle. It encodes two bits per symbol which helps to reduce BER. QPSK provides same data rate as BPSK for half the bandwidth needed by BPSK. But the complexity of transmitters and receivers is the major problem in QPSK.

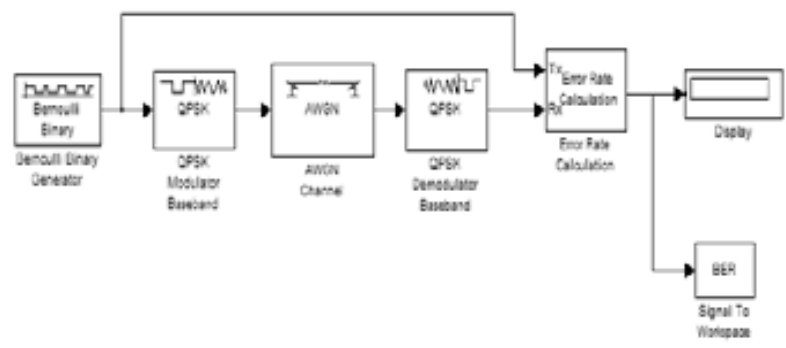

Fig.7: QPSK Simulink Model

Figure.7 shows simulink Model for Quadrature Phase Shift Keying. In general the MPSK signal is given by

$$
S_{n}(t)=\sqrt{\frac{2 E_{s}}{T_{s}}} \cos \left(2 \pi f_{c} t+\frac{2 \pi n}{M}\right)
$$

for QPSK technique $\mathrm{M}=4$ and $\mathrm{n}=1,2,3,4$.

The above equation with additional phase of ${ }^{\frac{\pi}{4}}$ can be written as

$$
\begin{gathered}
S_{n}(t)=\sqrt{\frac{2 E_{s}}{T_{s}}} \cos \left(2 \pi f_{c} t+\frac{2 \pi n}{M}+\frac{\pi}{4}\right) \\
S_{n}(t)=\sqrt{\frac{2 E_{s}}{T_{s}}}\left(\begin{array}{c}
\left.\left.\cos 2 \pi f_{c}\right) \cos \left(\frac{2 \pi n}{M}+\frac{\pi}{4}\right)-\sin \left(2 \pi f t_{c}\right) \sin \left(\frac{2 \pi n}{M}+\frac{\pi}{4}\right)\right) \\
\cos \left(2 \pi f_{c} t\right) \text { and } \sin \left(2 \pi f_{c} t\right)
\end{array}\right.
\end{gathered}
$$

in equation (9) represents two signals that are orthogonally separated. The In Phase and Quadrature components are given by

$$
\begin{aligned}
\varphi_{1}(t) & =\sqrt{\frac{2 E_{s}}{T_{s}}} \cos \left(2 \pi f_{c} t\right) \rightarrow \text { in phase component } \\
\varphi_{1}(t) & =\sqrt{\frac{2 E_{s}}{T_{s}}} \sin \left(2 \pi f_{c} t\right) \rightarrow \text { quadrature component }
\end{aligned}
$$

So it consists of 4 constellation points given by

$$
\left( \pm \sqrt{\frac{E_{s}}{2}} \pm \sqrt{\frac{E_{s}}{2}}\right)
$$

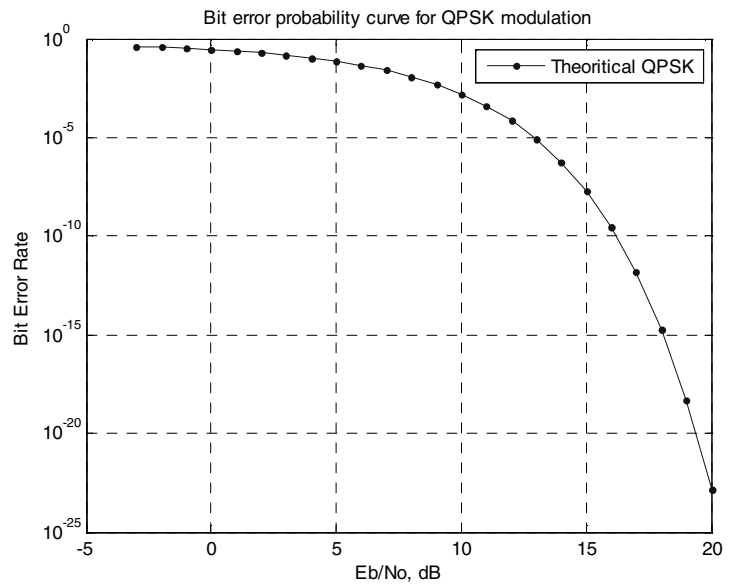

Fig. 8: Bit error rate probability for QPSK over AWGN

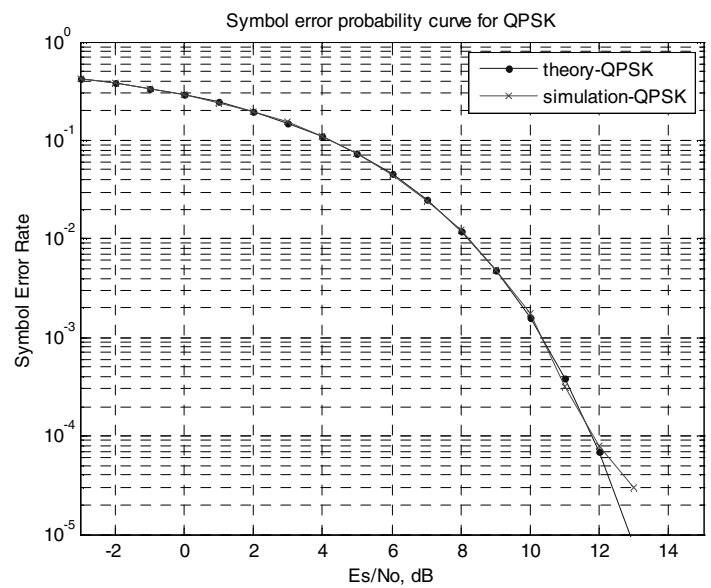

Fig.9: Comparative analysis of QPSK over AWGN Channel using MATLAB Simulink. 
Figure. 8 and 9 shows the theoritical and simulated bit error rate probability for QPSK over AWGN channel using MATLAB Simulink.

By substituting $\mathrm{M}=4$ we obtain the value of $\mathrm{P}_{\mathrm{s}}$ for QPSK. BER for BPSK is same as that of QPSK except channel bandwidth. It uses only half of the channel bandwidth and it transmits information at twice the bit rate of a BPSK system [5]. So it is mostly used in practice. The advantage of QPSK modulation is reduced bandwidth with increase in number of bits per symbol and disadvantage is that BER increases with increase in number of bits per symbol.

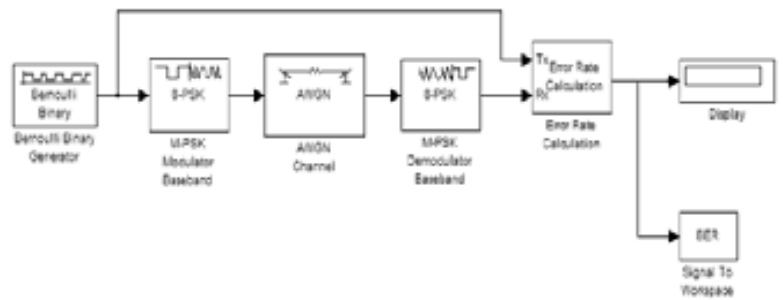

Fig.10: 8PSK Simulink Model

The 8PSK can be obtained by substituting $\mathrm{M}=3$ and is also known as Octa Phase Shift Keying. The Bandwidth for OPSK is fb/3. Fig. 11 shows the probability of error Vs $\mathrm{Eb} / \mathrm{No}$ for OPSK.

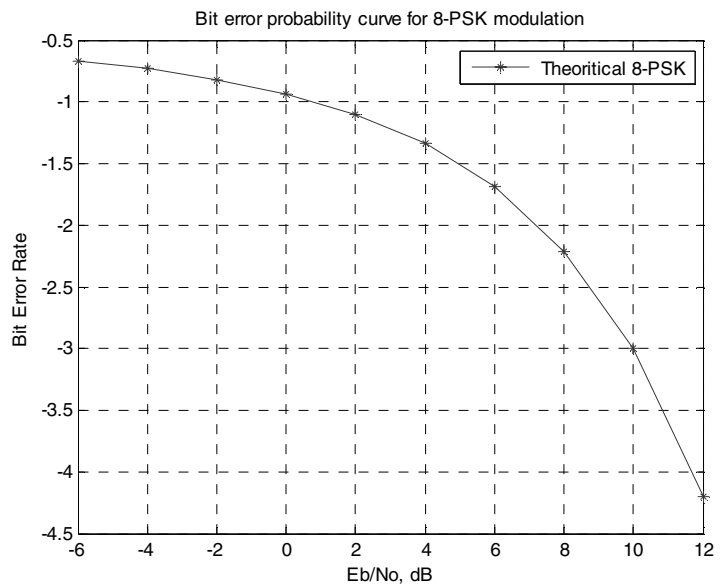

Fig.11: Bit error rate probability for 8-PSK over AWGN

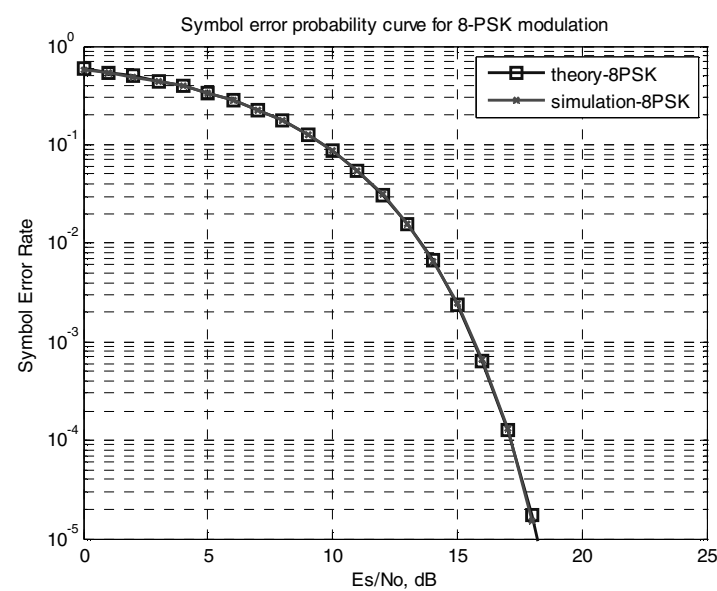

Fig.12: Comparative analysis of 8-PSK over AWGN Channel using MATLAB Simulink.

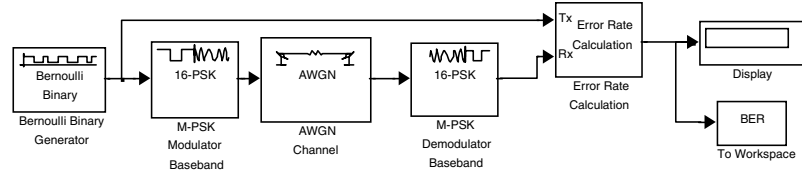

Fig: 13:16-PSK Simulink Model

Figure 13 shows the simulink model for 16-PSK and figure 14 and 15 shows the Theoritical and Simulated Bit Error Rates over AWGN Channel.

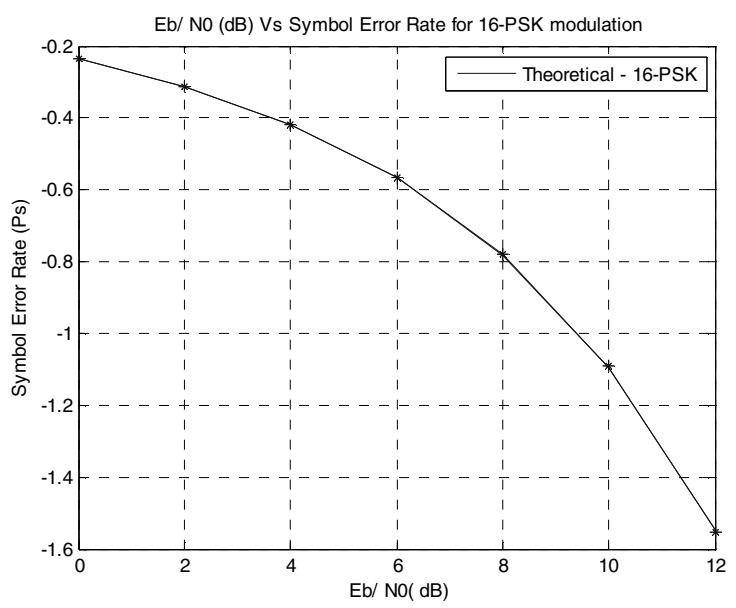

Fig.14: Bit error rate probability for 16-PSK over AWGN

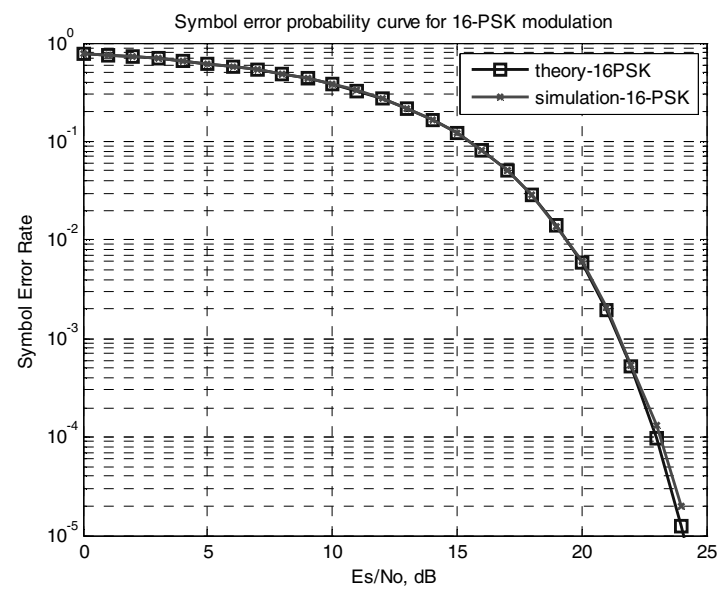

Fig.15: Comparative analysis of 16-PSK over AWGN Channel using MATLAB Simulink.

The 16-PSK is also known as Hexa phase shift keying. The Bandwidth for 16- PSK is fb/4. In this modulation the probability of errors and number of bits per symbols increases with the value of $\mathrm{M}$. The constellation points come closer as the distance between them decreases.

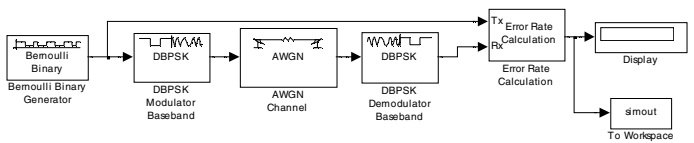

Fig.16:32-PSK Simulink Model 
Figure.16 shows the Simulink Model for 32-PSK and figure 17 and 18 shows the comparative analysis of theoritical and simulated bit error rates.

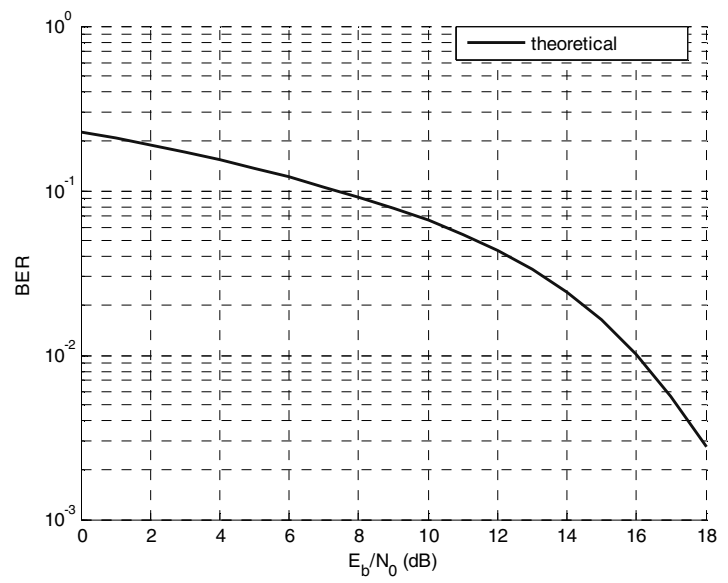

Fig.17: Bit error rate probability for 32PSK over AWGN

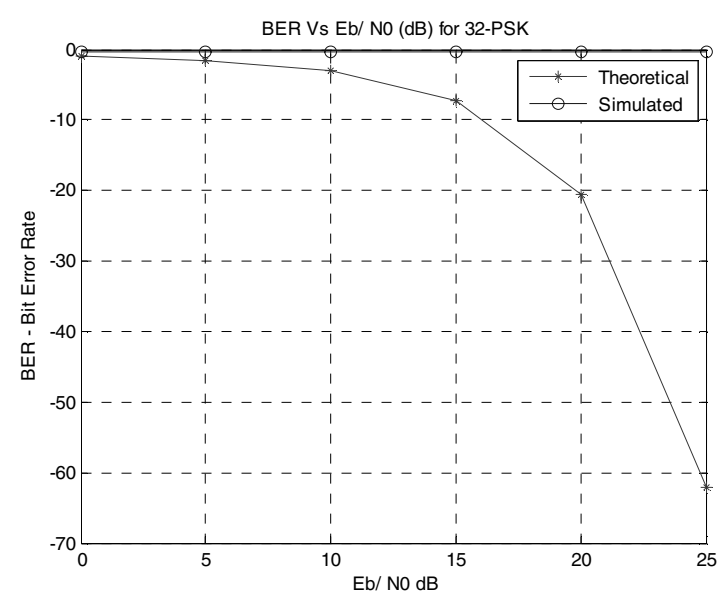

Fig.18: Comparative analysis of 32-PSK over AWGN Channel using MATLAB Simulink

DBPSK and DQPSK simulink model, theoritical and simulated bit error rate performances are shown in figure $19,20,21,22,23$ and 24.

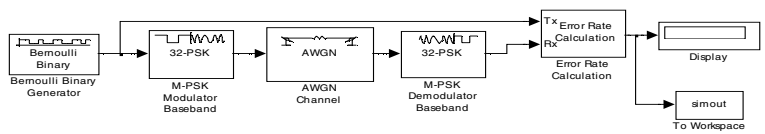

Fig.19: DBPSK Simulink Model

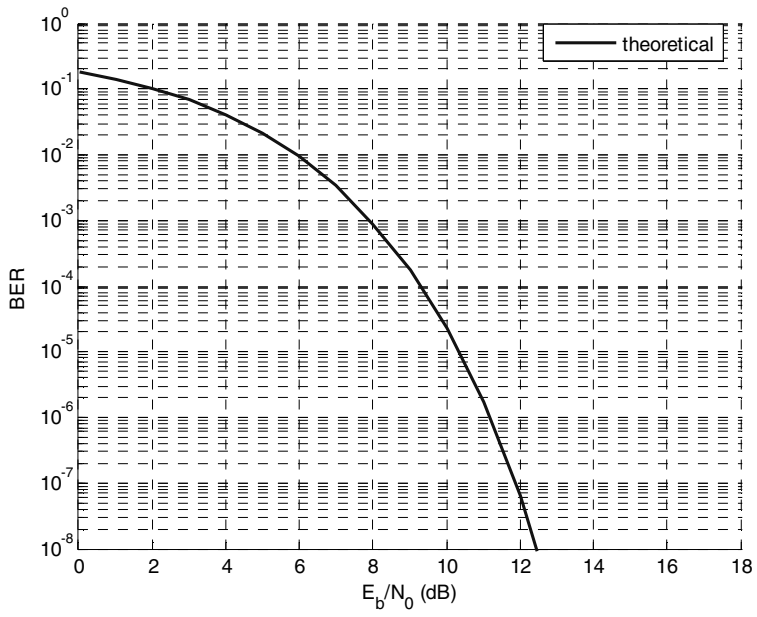

Fig.20: Bit error rate probability for DBPSK over AWGN

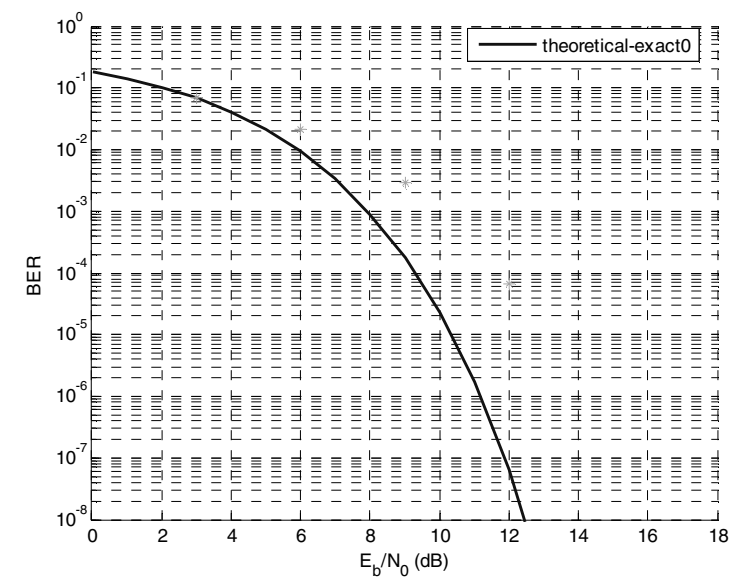

Fig. 21: Comparative analysis of DBPSK over AWGN Channel using MATLAB Simulink

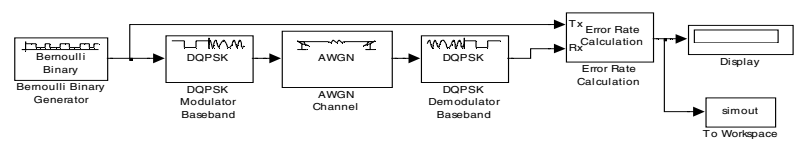

Fig.22: DQPSK Simulink Model

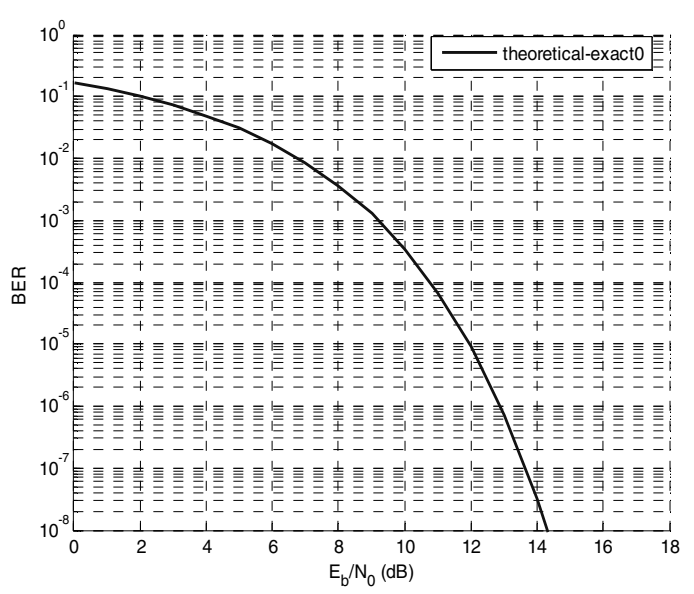

Fig.23: Bit error rate probability for DQPSK over AWGN 


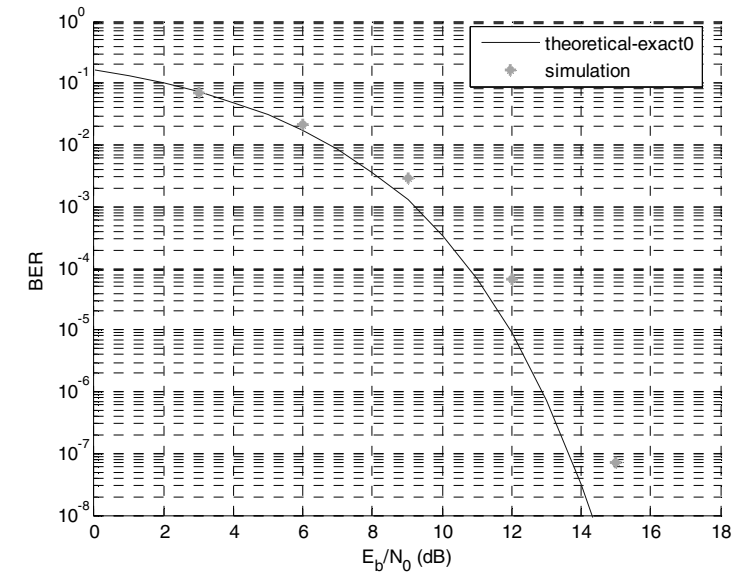

Fig.24: Comparative analysis of DQPSK over AWGN Channel using MATLAB Simulink

\section{Quadrature Amplitude Modulation (QAM)}

QAM transmits message by changing certain parameters of the carrier signal. In Quadrature Amplitude Modulation, amplitude is allowed to vary with phase,it is combination of ASK and PSK.But, implementation of this task is quite different. Unlike other techniques it Use s two quadrature carrier signals which are out of phase with each other by 90degrees. It transmits information by changing the amplitude of two carrier signals using ASK modulation technique. QAM can also be extended to M-QAM as 16QAM, 32QAM, 64QAM [2]. M-ary QAM modulation is more efficient than BPSK and QPSK.

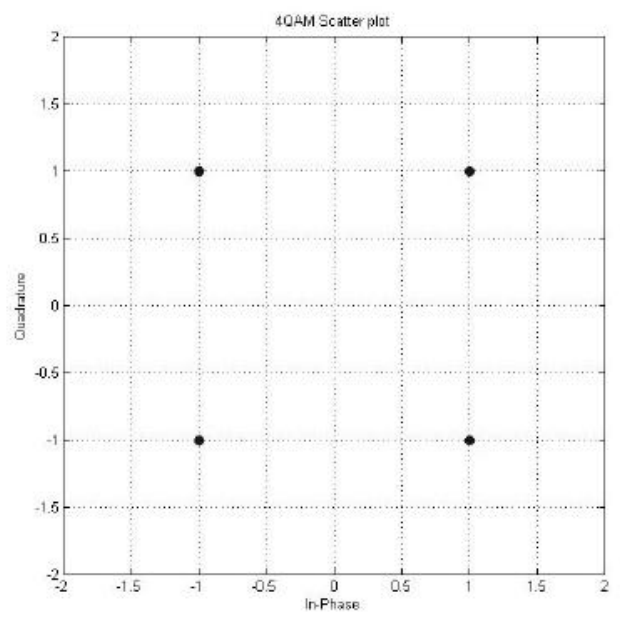

Fig.25: QAM Constellation

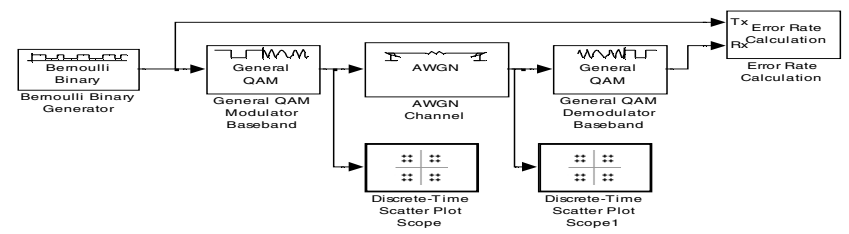

Fig.26: Simulink based M-ary QAM

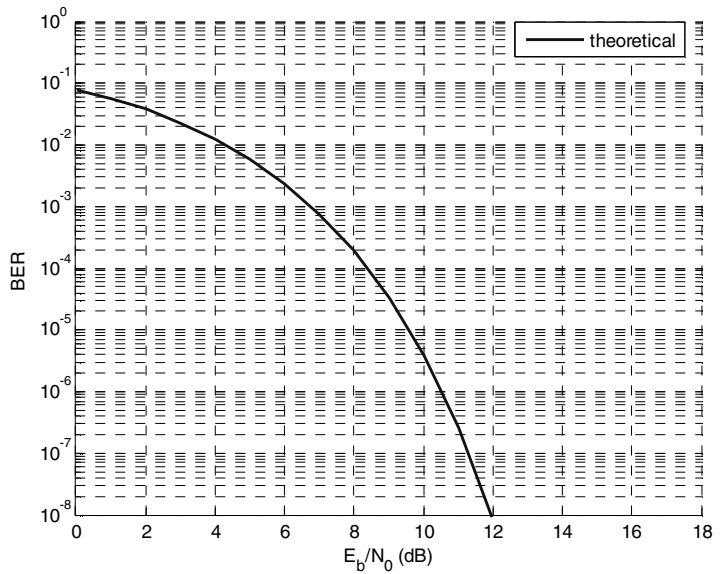

Fig.27: Bit error rate probability for 4QAM over AWGN

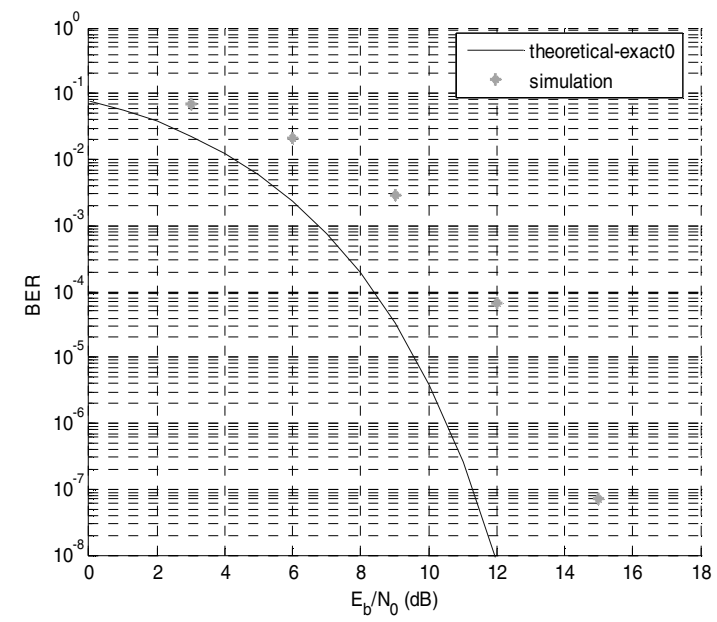

Fig.28: Comparative analysis of 4QAM over AWGN Channel using MATLAB Simulink

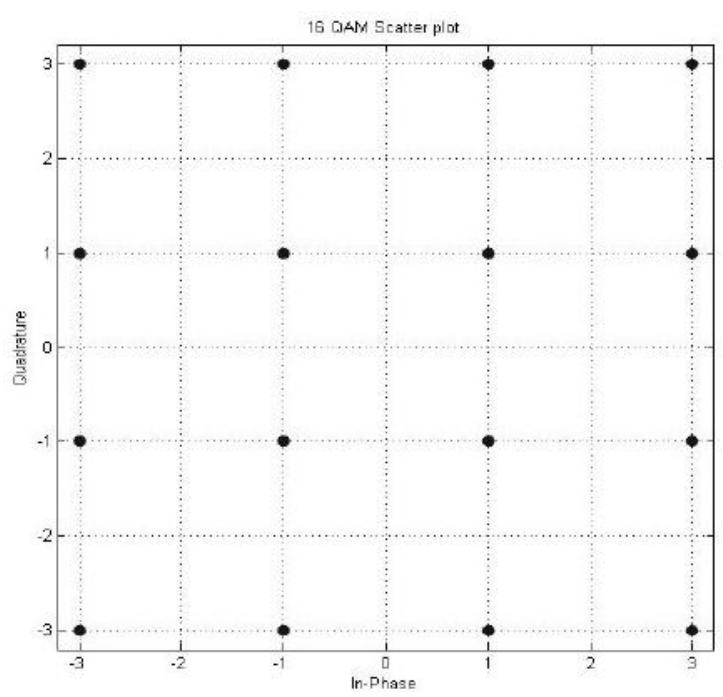

Fig.29:16-QAM Constellation

Similarly in other variations 16QAM four bits per symbol and symbol rate is one fourth of bit rate. 64QAM 
six bits per symbol and its rate is one sixth of bit rate.Additive White Gaussian Noise (AWGN) Channel is widely used to analyse the modulation schemes in communication systems [3]. AWGN channel just adds a Gaussian noise to the signal passing through it without any loss of amplitude and phase distortion of frequency components. In this the received signal $r(t)$ is sum of $s(t)$ transmitted signal and White Gaussian noise $\mathrm{n}(\mathrm{t})$

$$
r(t)=s(t)+n(t)
$$

$n(t)$ is sample function of Additive White Noise with probability density function(Pdf) and power spectral density as $N(f)$.

$$
N(f)=\frac{N_{0}}{2}-\infty<f<\infty
$$

Here No is noise power density, an example of radio signal with AWGN noise signal is shown below with SNR of $20 \mathrm{~dB}$.

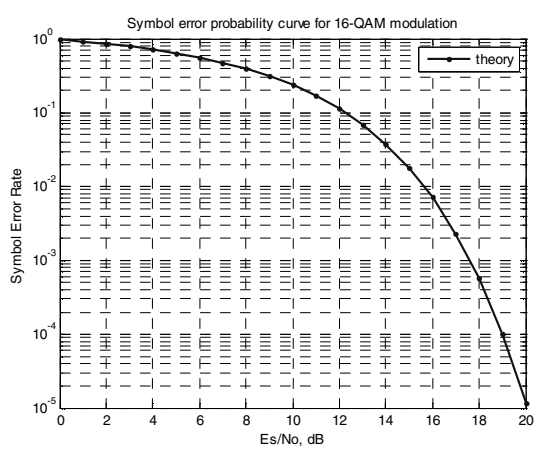

Fig.30: Bit error rate probability for 16QAM over AWGN

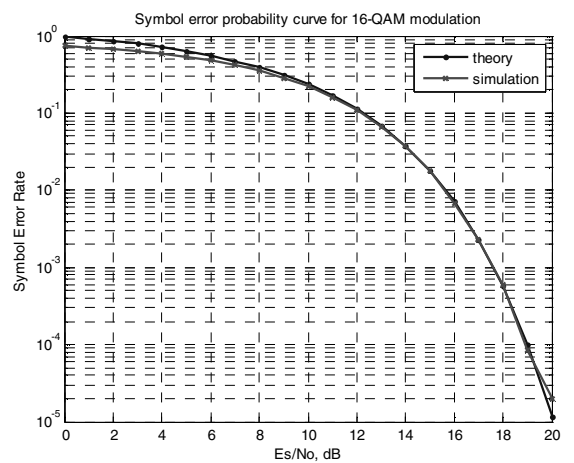

Fig.31:Comparative analysis of 16QAM over AWGN Channel using MATLAB Simulink

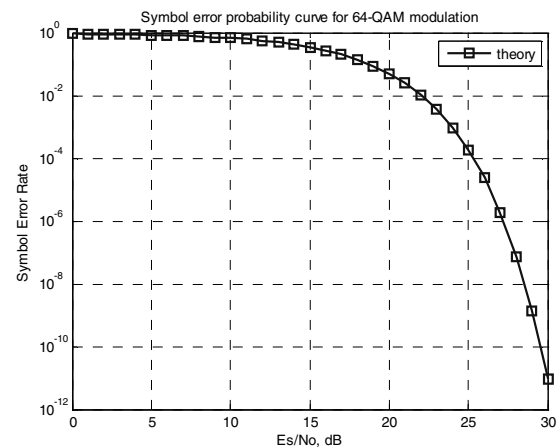

Fig.32:Bit error rate probability for 64QAM over AWGN

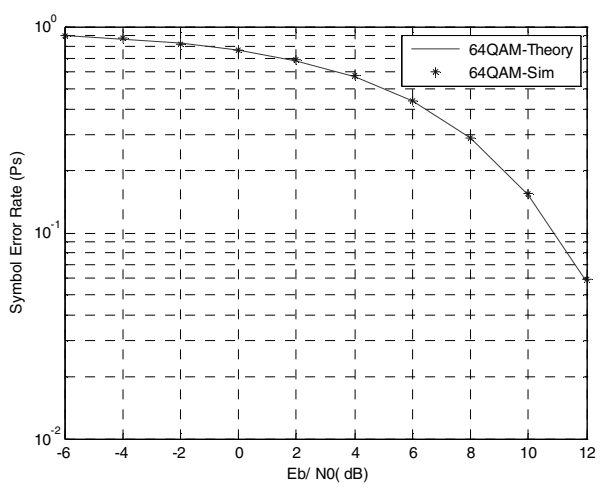

Fig.33: Comparative analysis of 64QAM over AWGN Channel using MATLAB Simulink

Theoretical BER for various linear modulations as shown in Table. 1

\begin{tabular}{|c|c|c|}
\hline Modulation & $\begin{array}{l}\text { Detection } \\
\text { Method }\end{array}$ & Bit Error Rate $\left(P_{b}\right)$ \\
\hline BPSK & Coherent & $0.5 \operatorname{erfc}\left(\sqrt{\frac{E_{b}}{N_{0}}}\right)$ \\
\hline QPSK & Coherent & $0.5 \operatorname{erfc}\left(\sqrt{\frac{E_{b}}{N_{0}}}\right)$ \\
\hline M-PSK & Coherent & $\frac{1}{m} \operatorname{erfc}\left(\sqrt{\frac{m E_{b}}{N_{0}}} \sin \left(\frac{\pi}{M}\right)\right)$ \\
\hline $\begin{array}{c}\text { M- } \\
\text { QAM(m=even) }\end{array}$ & Coherent & $\frac{2}{m}\left(1-\frac{1}{\sqrt{M}}\right) \operatorname{erfd}\left(\sqrt{\frac{3 m E_{b}}{2(M-1) N}}\right.$ \\
\hline D-BPSK & Non- Coherent & $0.5 e^{-\frac{E_{b}}{N_{0}}}$ \\
\hline D-QPSK & Non- Coherent & $\begin{array}{l}Q_{1}(a, b)-0.5 I_{0}(a b) e^{-0.5\left(a^{2}-\right.} \\
\text { where } a=\sqrt{\frac{2 E_{b}}{N_{0}}\left(1-\frac{1}{\sqrt{2}}\right.} \\
\quad b=\sqrt{\frac{2 E_{b}}{N_{0}}\left(1+\frac{1}{\sqrt{2}}\right)} \\
\quad \begin{array}{l}Q_{1}(a, b)=\text { MarcumQ } \\
I_{0}(\text { ab })=\text { ModifiedBessel }\end{array} \\
\text { function }\end{array}$ \\
\hline
\end{tabular}

Table 1: Theoretical BER over AWGN for various modulation techniques 


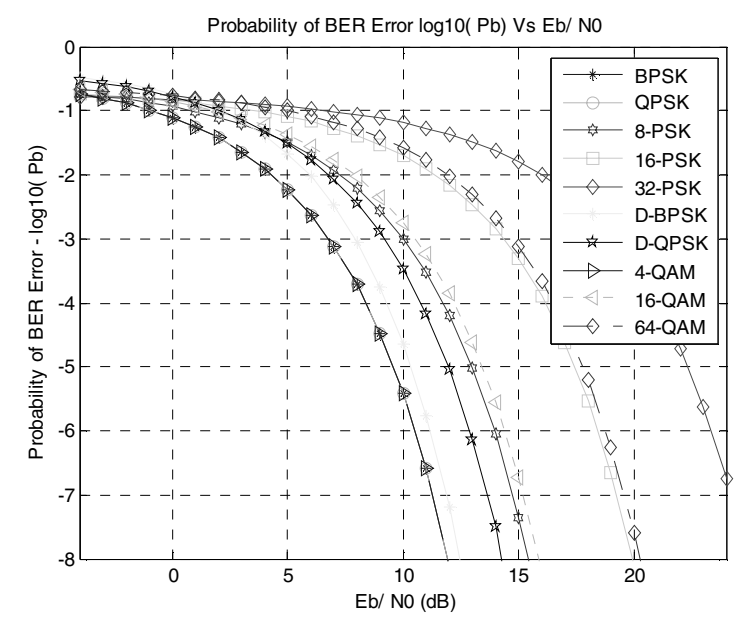

Fig.34: Comparative analysis of bit error rate probability for various modulation schemes over AWGN.

Fig.34 shows the comparison between BPSK, QPSK, 8PSK, 16-PSK, 32PSK, DBPSK, DQPSK, 4QAM, 16QAM and 64 QAM modulation schemes. The probability of error $\mathrm{Vs} \mathrm{Eb} / \mathrm{No}$ for the above mentioned digital modulation techniques are obtained graphically by using MATLAB simulation.32PSK gives better results than previous modulation techniques[4].

\section{SIMULATION RESULTS}

The results of Bit Error Rate performance of M-ary PSK for $\mathrm{M}=2,4,8,16$ and 32 ,M-aryQAM for $M=4,16$ and 64 , DBPSK and DQPSK obtained using MATLAB SIMULINK Communication Toolbox. The overall comparative analysis of theoretical and simulated curves for BER vs Eb/ No (signal to noise ratio)for BPSK,QPSK,8PSK, 16PSK,32PSK,DBPSK,DQP SK,4QAM,16QAM and 64QAM over AWGN channel are given in Fig.34.

\section{CONCLUSIONS}

In this paper the mathematical analysis and simulations using Matlab shows that the Bit Error Rate for the M-ary PSK, DBPSK,DQPSK and QAM modulation techniques decrease monotonically with increasing values of $\mathrm{SNR}(\mathrm{Eb} / \mathrm{N} 0)$. In QPSK system data transmission rate is twice the bit rate of a BPSK system . 8-PSK uses one third of the BW of BPSK and it transmits message at thrice the bit rate of a BPSK system that is clearly observed from the simulation curves. It is clearly observed that as the number of number of $M$ increases, the error probability also increases over AWGN channel. Thus these modulations exhibit higher error-rates. Increasing the data rate will increase the SNR, however, increasing $\mathrm{Rb}$ (Bit rate in bits /second) will also cause more noise, since more bits are packed closer and sent through the channel. So, we cannot increase SNR by simply increasing $\mathrm{Rb}$.

\section{FUTURE SCOPE}

The M-ary PSK can be implemented further by increasing $M$ value by using more number of bits per symbol in both MATLAB and Simulink.

\section{REFERENCES}

[1] Harjot Kaur,Bindiya Jain, Amit Verma."Comparative Performance Analysis of M-ary PSK Modulation Schemes using Simulink," IJECT Vol. 2, Issue 3, Sept. 2011.

[2] Xiaolong Li, Indiana State University."Simulink-based Simulation of Quadrature Amplitude Modulation (QAM) System",Proceedings of The 2008 IAJC-IJME International Conference ISBN 978-1-60643-379-9.

[3] Sklar, B."Digital Communications: Fundamentals and Applications", Prentice-Hall, 2nd Edition, pp. 30-33,2001.

[4] MATLAB Help Documents, Communications Toolbox.2009.

[5] Ho, W. S., "Adaptive modulation (QPSK, QAM),"www.intel.com/netcomms/technologies/wimax/303 788.pdf, accessed on December 30, 2007. 